\title{
Fluctuation measurements on the Wendelstein 7-AS stellarator by means of repetitive lithium laser blow-off
}

\author{
M Bruchhausen ${ }^{1}$, R Burhenn ${ }^{2}$, M Endler $^{2}$, G Kocsis $^{3}$, A Pospieszczyk ${ }^{1}$, \\ S Zoletnik $^{3}$ and the W7-AS Team ${ }^{2}$ \\ ${ }^{1}$ Institut für Plasmaphysik, Forschungszentrum Jülich GmbH EURATOM Association, Trilateral \\ Euregio Cluster, 52425 Jülich, Germany \\ ${ }^{2}$ Max-Planck-Institut für Plasmaphysik, EURATOM Association, 17491 Greifswald, Germany \\ ${ }^{3}$ KFKI-Research Institute for Particle and Nuclear Physics, EURATOM Association, PO Box 49, \\ 1525 Budapest, Hungary \\ E-mail: matthias.bruchhausen@free.fr
}

Received 7 October 2003

Published 12 February 2004

Online at stacks.iop.org/PPCF/46/489 (DOI: 10.1088/0741-3335/46/3/005)

\begin{abstract}
We present a method of beam emission spectroscopy (BES) using an atomic $\mathrm{Li}$ beam that is generated by means of laser blow-off for the investigation of electron density fluctuations in the edge and scrape-off layer plasmas of fusion devices. We discuss the operational limits of this technique and compare them to those of other atomic $\mathrm{Li}$ beam diagnostics.

Furthermore, we apply this method to different Wendelstein 7-AS discharges and study the influence of the line integrated electron density and the direction of the main magnetic field on the fluctuations inside and outside the last closed flux surface, and especially on their movement.

The information we obtain on the influence of the main magnetic field on the fluctuations gives some insight into the possible origin of certain features of their spatial structure.
\end{abstract}

\section{Introduction}

Anomalous transport in fusion plasmas is dominated by correlated fluctuations of the particle densities and temperatures and the electrical and magnetic fields [1-3]. Therefore, many diagnostics have been developed to investigate fluctuation phenomena in core as well as in edge and scrape-off layer (SOL) plasmas [4].

In the special case of the electron density $n_{\mathrm{e}}$ and its fluctuations $\tilde{n}_{\mathrm{e}}$ atomic Li beams have proven to be a powerful tool in SOL and edge plasmas. Up to now mainly thermal $[5,6]$ and high energy [7-9] Li beams have been used for this purpose. However, both methods have their specific drawbacks: the small velocity of the atoms in a thermal beam restricts its use to the SOL 
and lower densities whereas the high energy beam has a spatial resolution which for certain plasma conditions is larger than the correlation lengths of the electron density fluctuations [9].

The Li laser blow-off technique has proven its usefulness for the measurement of $n_{\mathrm{e}}$-profiles $[10,11]$. Its application to fluctuation measurements has been proposed [12] but was never realized on fusion devices before.

In section 2, we explain the principle of this method and discuss its limitations in comparison to thermal and high energy Li beams.

The experimental realization of this diagnostic on the Wendelstein 7-AS (W7-AS) stellarator is presented in section 3 .

The data evaluation was done by means of correlation functions. This is a commonly used method in fluctuation analysis and we will only give a short description. As the intensity of the beam varies strongly with time, the separation between variations of the beam intensity and fluctuation phenomena is essential. Both subjects are treated in section 4 .

In section 5, we present some experimental results concerning a change of the fluctuation behaviour around the last closed flux surface (LCFS) and its dependence on the direction of the toroidal magnetic field and in section 6, we discuss these results.

\section{Measurement principle}

Laser blow-off has been discussed as a source for $1-10 \mathrm{eV}$ atoms for the first time in the seventies [13-15]. A laser blow-off source consists of a laser and a substrate which is transparent for the laser wavelength. The substrate is coated with a layer (typically $0.1 \mu \mathrm{m}$ ) that contains the material one wants to inject and which must absorb the laser beam. If a laser pulse is shot through the substrate onto the coating, the energy of the pulse will be absorbed in the layer and an expanding plasma cloud is formed. Because of the reflection of the atoms on the surface of the substrate, the atoms get a velocity component that is directed perpendicularly to the surface. In the simplest case, the velocity distribution of the atoms can be described as a constant velocity with a superposed thermal distribution in the frame of the centre of mass [16]. The exact velocity distribution depends on the thickness of the coating and the energy density of the laser pulse, and can contain several components (atoms, clusters) [17-19]. For measurements of the electron density, however, it is essential that the atomic beam contains no clusters, as those act as a source of atoms within the beam.

If such a beam is injected into a fusion plasma, the atoms are excited and finally ionized by the plasma electrons. If the excited states are populated from the lower states and not from free plasma electrons, the interaction between the plasma and the atoms can be described by a corona model (excitation and ionization by electrons, relaxation by radiation).

According to [20], only bound levels above the level

$$
k=\left(7 \times 10^{24} \frac{Z^{6}}{n_{\mathrm{e}}} \sqrt{\frac{T_{\mathrm{e}}}{E_{\text {ion, hydrogen }}}}\right)^{2 / 17}
$$

are in equilibrium with the continuum (Saha equation). Here, $Z$ is the charge number ( 3 for Li), and $E_{\text {ion,hydrogen }}$ is the ionization energy of hydrogen $(13.6 \mathrm{eV})$. The electron density $n_{\mathrm{e}}$ is in $\mathrm{m}^{-3}$ and the electron temperature $T_{\mathrm{e}}$ is in electronvolts. In those discharges investigated here, typical values at $r_{\text {eff }}=14 \mathrm{~cm}$ are $n_{\mathrm{e}} \approx 1.5 \times 10^{19} \mathrm{~m}^{-3}$ and $T_{\mathrm{e}} \approx 200 \mathrm{eV}$. Therefore, levels below $k=11$ are not in equilibrium with the continuum.

The Einstein coefficient for the $\mathrm{Li}$ resonance line $(2 \mathrm{p} \rightarrow 2 \mathrm{~s})$ observed here is: $A=3.72 \times 10^{7} \mathrm{~s}^{-1}$. With a velocity of the atoms of $v_{\mathrm{a}} \leqslant 10^{4} \mathrm{~m} \mathrm{~s}^{-1}$ that means that the atoms travel less than $1 \mathrm{~mm}$ during the time interval $1 / A$ and the plasma parameters can be 
considered as constant. Therefore, one can assume a corona model in the case of the blow-off Li beam.

Hence, one can derive an expression for the electron density profile [11]:

$$
n_{\mathrm{e}}(r)=\frac{\bar{v}_{\mathrm{a}} I_{\mathrm{a}}(r)}{\left\langle\sigma_{12} v_{\mathrm{e}}\right\rangle \int_{r}^{r_{0}}\left(\left\langle\sigma_{\mathrm{I}} v_{\mathrm{e}}\right\rangle /\left\langle\sigma_{12} v_{\mathrm{e}}\right\rangle\right) I_{\mathrm{a}}\left(r^{\prime}\right) \mathrm{d} r^{\prime}}
$$

In this equation, $\bar{v}_{\mathrm{a}}$ is the average velocity of the atoms in the beam and $I_{\mathrm{a}}$ is the intensity of the light emitted by the atoms. The integration has to be executed from the point of observation $r$ to the point $r_{0}$ where all atoms are ionized. The centre of the coordinate system is in the plasma centre and the atoms propagate from $r>0 \rightarrow r=0\left(\bar{v}_{\mathrm{a}}<0\right)$.

$\left\langle\sigma_{12} v_{\mathrm{e}}\right\rangle$ and $\left\langle\sigma_{\mathrm{I}} v_{\mathrm{e}}\right\rangle$ are the rate coefficients for electron impact excitation and ionization, respectively. They have been calculated with the cross sections $\sigma_{12}$ from [21] and $\sigma_{\mathrm{I}}$ from [22] under the assumption of a Maxwellian velocity distribution of the plasma electrons. Here we take into account only the ionization from the ground state. It has been shown that the influence from the ionization of excited atoms is mostly compensated by the increase of $\left|\bar{v}_{\mathrm{a}}\right|$ towards the plasma centre [23]. This apparent acceleration of the beam is caused by the fact that the slower atoms are ionized closer to the plasma edge. The line that is observed here is the resonance line of $\mathrm{Li}$ at $\lambda=670.8 \mathrm{~nm}$, which corresponds to the transition $2 \mathrm{p} \rightarrow 2 \mathrm{~s}$.

For electron temperatures $T_{\mathrm{e}}$ in the range between 10 and $200 \mathrm{eV}$, the ratio of the rate coefficients $\left\langle\sigma_{\mathrm{I}} v_{\mathrm{e}}\right\rangle /\left\langle\sigma_{12} v_{\mathrm{e}}\right\rangle$ depends only weakly on $T_{\mathrm{e}}$ and therefore on $r$. Hence, one may simplify equation (2) to:

$$
n_{\mathrm{e}}(r)=\frac{\bar{v}_{\mathrm{a}} I_{\mathrm{a}}(r)}{\left\langle\sigma_{\mathrm{I}} v_{\mathrm{e}}\right\rangle \int_{r}^{r_{0}} I_{\mathrm{a}}\left(r^{\prime}\right) \mathrm{d} r^{\prime}}
$$

This means that it is possible to determine the profile of the absolute electron density by measuring the relative emission profile up to the point of complete ionization.

In the following, we will discuss the parameter range in which the Li laser blow-off can be used for fluctuation measurements.

\subsection{Temperature range}

Equation (3) is only valid in the temperature range $10 \mathrm{eV} \leqslant T_{\mathrm{e}} \leqslant 200 \mathrm{eV}$. This condition is normally satisfied in the SOL and edge of fusion plasmas.

If this condition is not fulfilled, one has to know the temperature profile $T_{\mathrm{e}}(r)$ to calculate profile of the electron density $n_{\mathrm{e}}(r)$.

\subsection{Penetration depth}

The ionization of the atoms by electron impact restricts the applicability of this technique to the plasma edge.

One can characterize the penetration depth by the point $r_{1 / \mathrm{e}}$ where the number of atoms in the beam has decreased to $1 / \mathrm{e}$ of the initial amount of atoms. If the condition for the temperature range is fulfilled, $\left\langle\sigma_{\mathrm{I}} v_{\mathrm{e}}\right\rangle$ only depends weakly on $T_{\mathrm{e}}$ and therefore on $r$ and one can derive a criterion for the penetration depth $r_{1 / \mathrm{e}}$ :

$$
\int_{r_{1 / \mathrm{e}}}^{\infty} n_{\mathrm{e}}\left(r^{\prime}\right) \mathrm{d} r^{\prime} \approx \frac{\left|\bar{v}_{\mathrm{a}}\right|}{\left\langle\sigma_{\mathrm{I}} v_{\mathrm{e}}\right\rangle}
$$

For our laser blow-off system $\left(\bar{v}_{\mathrm{a}} \approx 10^{4} \mathrm{~m} \mathrm{~s}^{-1}\right)$ and $\operatorname{Li}\left(\left\langle\sigma_{\mathrm{I}} v_{\mathrm{e}}\right\rangle \approx 6 \times 10^{-14} \mathrm{~m}^{3} \mathrm{~s}^{-1}\right)$, one obtains a penetration depth of $\int n \mathrm{~d} r=2 \times 10^{17} \mathrm{~m}^{-2}$. 
Table 1. Comparison of the three $\mathrm{Li}$ beams with regard to fluctuation measurements. The confinement regions where the diagnostics can be used are given for medium size experiments as TEXTOR or W7-AS.

\begin{tabular}{llll}
\hline & Thermal & Blow-off & High energy \\
\hline Energy & $50-100 \mathrm{meV}$ & $5-10 \mathrm{eV}$ & $50 \mathrm{keV}$ \\
Penetration depth & $2 \times 10^{16} \mathrm{~m}^{-2}$ & $2 \times 10^{17} \mathrm{~m}^{-2}$ & $10^{19} \mathrm{~m}^{-2}$ \\
Plasma region & $\mathrm{SOL}$ & Edge plasma & Core plasma \\
Radial resolution & $0.2 \mathrm{~mm}$ & $2 \mathrm{~mm}$ & $10-20 \mathrm{~mm}$ \\
Length of pulse & Continuous & $100-300 \mu \mathrm{s}$ & Continuous \\
Frequency resolution & Very high & $10-3 \mathrm{kHz}$ & Very high \\
\hline
\end{tabular}

\subsection{Spatial resolution}

If the beam encounters a spatial variation of the electron density, it takes a certain time for the atoms to reach equilibrium between the excited states again. For a thermal $\mathrm{Li}$ beam this time has been determined to be of the order of $\tau_{E}=0.2 \mu \mathrm{s}$ [24]. As the atoms of the thermal and blow-off beam are both much slower than the plasma electrons and since other collisions can be neglected in both cases, the time to reach equilibrium is the same for both beams. For the laser blow-off beam, this leads to a spatial resolution of the order of $2 \mathrm{~mm}$.

Table 1 summarizes some properties of the three $\mathrm{Li}$ beams with regard to fluctuation measurements. As the correlation lengths in the SOL are typically of the order of 5-10 mm, one can see that from the atomic physics point of view, the blow-off beam is the best possible compromise between spatial resolution and penetration depth for the investigations of such structures.

\subsection{Temporal resolution}

Of course, a finite $\tau_{E}$ leads also to a restriction of the temporal resolution. However $1 / \tau_{E}=5 \mathrm{MHz}$ is fast enough to measure all edge fluctuation phenomena which are generally characterized by frequencies below $100 \mathrm{kHz}[29,30,24]$.

In general, the Nyquist frequency $v_{\mathrm{Ny}}$ that is half of the sampling rate [31] is a much larger constraint. In our case, the sampling rate was $1 \mathrm{MHz}$ and therefore sufficiently high for our purposes.

\subsection{Frequency measurements}

The laser blow-off system can only produce pulses of limited length. The exact length depends on the details of the set-up (e.g. the distance between the coated target and the plasma), but one can hardly obtain pulses that are longer than $T_{0}=100-300 \mu \mathrm{s}$. One can increase the statistics by using a repetitive system as the one presented in section 3 but the frequency resolution of the fluctuation spectra is limited to $\Delta v=1 / T_{0}$ which means $\Delta v=3-10 \mathrm{kHz}$. As fluctuation spectra are usually characterized by broad spectra rather than by strong lines, this is not necessarily a problem for such measurements, but it can be a major drawback if one is interested in phenomena with characteristic frequencies.

Accordingly, no phenomena with characteristic frequencies below $3-10 \mathrm{kHz}$ can be resolved.

\subsection{Velocity of the fluctuations}

If the velocity of the beam and the velocity of the fluctuations of the electron density in the direction of propagation of the beam are of similar magnitude, the interpretation of the signal 
becomes very difficult. One would have to separate the influence of the two movements on the light fluctuations.

In the case of a radial movement, especially the atoms of the thermal beam have a velocity that is similar to the velocity of the fluctuations of the electron density. This problem has been discussed in more detail and shown experimentally in [12].

The blow-off beam in contrast has a velocity that is significantly (at least one order of magnitude) larger than the radial velocity of the fluctuations and the velocity of the high energy beam is much larger than that.

This means that the radial velocity of the fluctuations of the electron density is neither a concern for the blow-off beam nor for the high energy beam but has to be considered for the thermal beam.

\subsection{Perturbation of the plasma by the Li beam}

As with the laser blow-off material is injected into the plasma, it is important to study if this injection does disturb the plasma significantly. First, the perturbation of the plasma density $n_{\mathrm{e}}$ is discussed and then the perturbation of the plasma temperature $T_{\mathrm{e}}$.

2.7.1. Perturbation of the electron density $n_{\mathrm{e}}$. It is difficult to determine the number of atoms that are injected, as the opening cone of the beam is not well known and thus the fraction of atoms that are deposited on the walls of the vacuum vessel or the collimating slit (see section 3.1) cannot be calculated exactly. An upper estimate of the number of particles can, however, be obtained by calculating the number of particles in that part of the target coating that is ablated in a typical laser pulse. For a layer of $0.1 \mu \mathrm{m}$ and a laser spot diameter of $1.5 \mathrm{~mm}$, of the order of $10^{16} \mathrm{Li}$ atoms are ablated.

The ratio of translational to transverse velocity is approximately $5: 1$ [18]. That means that after travelling the distance between the target plate and the measurement volume, the beam has a diameter of $40 \mathrm{~cm}$. A slit in the path of the injection system (see section 3 ) collimates the beam to a size of $1 \mathrm{~cm} \times 10 \mathrm{~cm}$ in the measurement region. That means the number of particles injected into the plasma is reduced by a factor of $10 \mathrm{~cm}^{2}: 20 \mathrm{~cm} \times 20 \mathrm{~cm} \times \pi \approx 10: 1000$, which means that about $10^{14}$ atoms reach the plasma.

The real number of injected particles is probably lower, as a part of the atoms is ionized (the photon energy of the excimer laser $(4 \mathrm{eV})$ is close to the ionization energy of $\mathrm{Li}(5.4 \mathrm{eV})$ ). In addition, at the edges of the ablated region, the energy density on the target plate may be too small to atomize the target material completely, which would lead to the creation of small clusters or fragments.

Assuming that $10^{14}$ atoms are deposited in a volume of approximately $10 \times 1 \times 5 \mathrm{~cm}^{3}$ (toroidal $\times$ poloidal $\times$ radial) during a pulse of approximately $100 \mu$ s one obtains an influx of $10^{22} \mathrm{~s}^{-1} \mathrm{~m}^{-3}$. The ionized particles leave the deposition area with a velocity of approximately $1 \mathrm{~m}$ in $100 \mu \mathrm{s}$ [25], which means that they leave the deposition area in about $10 \mu \mathrm{s}$. So one can estimate the number of particles that are deposited during that time to be of the order of $10^{17} \mathrm{~m}^{-3}$. The electrons originating from the injected fluorine and chromium (which are components of the target material, see section 3) do not change this estimate. The number of injected particles is roughly the same as for lithium, and especially fluorine is ionized further in the plasma (the ionization energies are higher: $17.4 \mathrm{eV}$ for fluorine and $6.8 \mathrm{eV}$ for chromium).

We therefore estimate that the maximum number of particles deposited is of the order of $1 \%$ of the local plasma density and, hence, even less than the amplitude of the density fluctuations. 
2.7.2. Perturbation of the electron temperature $T_{\mathrm{e}}$. The ionization of the injected particles as well as their line radiation lead to a cooling of the plasma. For the confinement region, an upper limit for the power losses caused by these effects can be estimated with data from [26]: ionization of all injected atoms leads to power losses of the order of $150 \mathrm{~W}$ and the losses by line radiation are of the order of a few kilowatts. As the parallel thermal velocity of the electrons in the plasma is high compared to the velocity of the injected atoms and their mean free path is in excess of $10 \mathrm{~m}$, one may assume that inside the LCFS all electrons on the flux surface will contribute to the compensation of the energy losses caused by the injected atoms. In the flat top phase of a typical discharge (e.g. \#52772), the total heating power was $P_{\text {heat }}=400 \mathrm{~kW}$ and the total radiated power was $P_{\text {rad }}=30-40 \mathrm{~kW}$. In the stationary phase of such a discharge, the difference $P_{\text {heat }}-P_{\text {rad }}=350 \mathrm{~kW}$ must be transported by anomalous transport (turbulent convection) radially across the LCFS into the SOL and is thus available to compensate the local cooling. The total power losses caused by ionization and line radiation are less than $1 \%$ of the available power and, hence, negligible.

In the SOL, time dependent deposition, ionization and transport processes and the complex nature of this region make it a difficult task to obtain a meaningful estimate of a possible perturbation. Such a detailed analysis of all the processes is beyond the scope of this paper. However, in the past, laser blow-off measurements have been used to determine the electron density and temperature in the SOL by injection of LiF and C [27]. As no collimator slit but a thicker target layer and spot diameter were used, the amount of injected material (Li, F, C) was larger by a factor of $\approx 10^{2}$ than for the measurements presented here. The temperature profiles from these measurements were compared to those from other diagnostics (probes, He beam) and no cooling effect was found [28]. These experimental results show that the injection of a small amount of atoms - as during the measurements described here-does not lead to a significant cooling of the plasma even in the SOL.

\subsection{Signal to noise ratio}

The temporal and spatial resolution is not defined by the timescale of the atomic physics processes alone, but the detected photon flux also plays an equally important role. A rough estimate on the photon flux can be made the following way. The number of photons emitted by one injected $\mathrm{Li}$ atom is determined by the ratio of the excitation rate to the ionization rate, as a rough estimate this is about $R \approx 10$ [21,22]. The total number of injected atoms is about $N_{\text {tot }}=10^{14}$ (see section 2.7) and one observation channel observes about $\mathrm{d} l / L=1 / 20$ part of the beam penetration depth. The detection solid angle is approximately $\Omega=10^{-3}$, and the detection quantum efficiency is about $Q=0.01$. From these data, the total number of photons detected in one channel during the total length of one LBO pulse is:

$$
F=N_{\text {tot }} R \frac{\mathrm{d} l}{L} \Omega Q \approx 10^{9}
$$

The length of the LBO pulse is about $100 \mu \mathrm{s}$, therefore about $10^{8}$ photons can be detected in the $1 \mu$ s time resolution. The photon statistical $S / N$ ratio from this is $10^{3}$. Even if the number of injected particles is overestimated by two orders of magnitude in section 2.7 , the $S / N$ ratio is still $10^{2}$, which is comparable to or less than the typical amplitude of the density fluctuations in the plasma $\left(10^{-2}-10^{-1}[9]\right)$. This estimated $S / N$ ratio is significantly higher than for a high energy Li beam [8].

We should emphasize, however, that our evaluation method using cross correlation functions (see section 4.1) is rather insensitive to uncorrelated noise, which would only show up as a clearly distinguishable peak in the autocorrelation function at time delay $\tau \approx 0$. 


\section{Experimental set-up}

The set-up that was used for the measurements consists of two parts, the injection and the observation system that are shown in figures $1(a)$ and $(b)$, respectively.

\subsection{Injection system}

For the injection, we used a XeCl excimer laser (Lambda Physik EMG 150) at $308 \mathrm{~nm}$ with a pulse duration of $18 \mathrm{~ns}$ and a pulse energy of $100 \mathrm{~mJ}$ on the target. The targets themselves were $50 \mathrm{~mm} \times 50 \mathrm{~mm}$ large quartz substrates which were coated with a $0.05 \mu \mathrm{m}$ Cr layer to absorb the laser energy and a $0.1 \mu \mathrm{m}$ layer of $\mathrm{LiF}$ on top.

A lens was used to collimate the laser beam onto the target. The typical diameter of the blow-off spot was 1-2 mm, depending on the position of the lens.

For the fluctuation measurements, it was essential to inject several times into the same discharge in order to increase the total measurement time and hence the statistics. Therefore, the

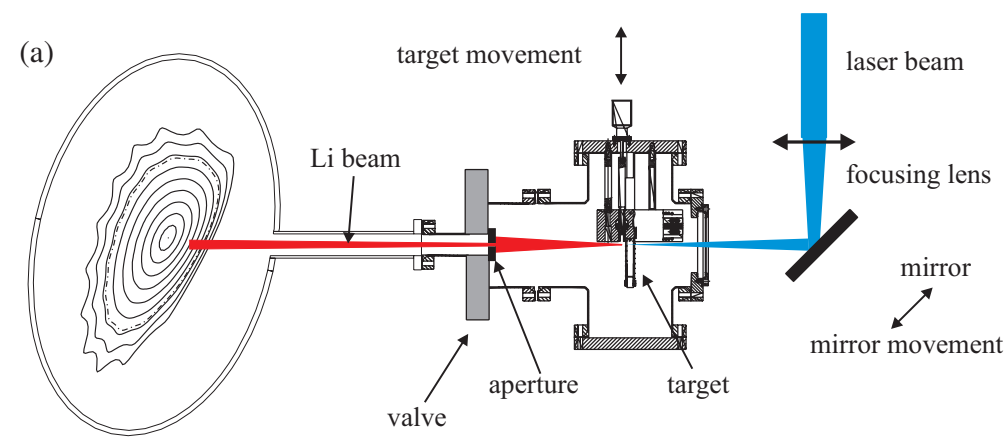

(b) to photomultiplier tubes

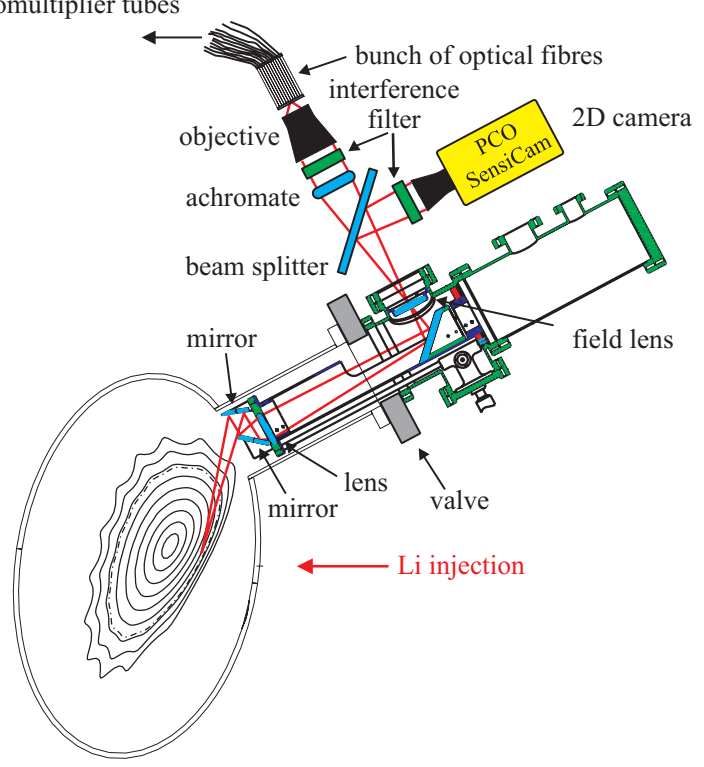

Figure 1. Representation of ( $a$ ) the injection system and $(b)$ the observation system used on W7-AS. 
last mirror together with the focusing lens and the target itself could be moved in perpendicular directions in order to scan a target with the laser spot at a frequency of $\approx 10 \mathrm{~Hz}$. The vacuum system contained up to 15 different targets so that an empty target could easily be replaced between two successive discharges.

The atomic beam itself was collimated by a slit to have a poloidal width of $\approx 10 \mathrm{~mm}$ on the location of the measurement which corresponds to the poloidal extension of the fluctuating cells. As the correlation length of fluctuations in toroidal direction is of the order of several metres [32], no collimation of the beam was necessary in that direction. In contrary, the use of the entire width of the beam in toroidal direction permitted us to maximize the signal intensity without introducing an error by averaging over several fluctuating cells.

\subsection{Observation system}

To observe the interaction volume between the Li beam and the plasma, we used the imaging system shown in figure $1(b)$. First the volume was imaged onto a field lens close to the vacuum window. This image was then observed simultaneously by a photomultiplier array with eight equally spaced channels covering radially $18 \mathrm{~mm}$ of the plasma and a two-dimensional CCD camera (PCO SensiCam). In both cases interference filters were used to reduce the background light. Both filters had a central wavelength of $\lambda_{0}=670.8 \mathrm{~nm}$. The filter used for the photomultipliers had a width of $\Delta \lambda=1.5 \mathrm{~nm}$ and the one in front of the camera a width of $\Delta \lambda=6.2 \mathrm{~nm}$.

The data from the photomultiplier array was sampled with $1 \mathrm{MHz}$ and used for the fluctuation measurements as explained in section 4. The camera pictures were integrated toroidally and could be used to relatively calibrate the photomultipliers as well as for the calculation of electron density profiles.

\section{Data evaluation}

\subsection{Correlation analysis}

The correlation function $\Phi_{f g}(\tau)$ of two time dependent functions $f(t)$ and $g(t)$ is given by:

$$
\Phi_{f g}(\tau)=\int_{-\infty}^{\infty} f(t) g(t+\tau) \mathrm{d} t
$$

In our case, the two functions $f(t)$ and $g(t)$ correspond to the time traces of two photomultiplier signals. To compare the correlation functions between several channels independently from their respective signal amplitudes, one can use the normalized correlation functions:

$$
\varphi_{f g}(\tau)=\frac{\Phi_{f g}(\tau)}{\sqrt{\Phi_{f f}(0) \Phi_{g g}(0)}}
$$

As the correlation function according to equation (6) and the crosspower spectrum

$$
K_{f g}(v)=F^{*}(v) G(v)
$$

are a pair of Fourier transforms, one can calculate the correlation functions by means of fast Fourier transformation (FFT) algorithms. In equation (8) $F(v)$ and $G(v)$ denote the Fourier transforms of $f(t)$ and $g(t)$ and the asterisk denotes the complex conjugate.

In the case of our diagnostic, the correlation function depends on the radial positions $r_{1}$ and $r_{2}$ of the two channels as well as on the time lag $\tau$. Instead of $\Phi_{f g}(\tau)$, we therefore write $\Phi\left(r_{1}, r_{2}, \tau\right)$. Hence, the normalized correlation function becomes:

$$
\varphi\left(r_{1}, r_{2}, \tau\right)=\frac{\Phi\left(r_{1}, r_{2}, \tau\right)}{\sqrt{\Phi\left(r_{1}, r_{1}, 0\right) \Phi\left(r_{2}, r_{2}, 0\right)}}
$$


To plot such a function, one has to choose one radial channel at fixed $r_{1}=$ const and can plot the correlation in dependence of $r_{2}$ and $\tau$. The fixed channel has to be specified for every plot.

From such a representation, one can read characteristic parameters of the fluctuations. An example for this is shown in figure 2. The elliptic shapes in the graphs correspond to levels of constant correlation. The radial correlation length is the $1 / \mathrm{e}$ folding length in radial direction as shown in figure $2(a)$. If one determines in a similar way the decay length in $\tau$-direction, one obtains the width $2 \tau_{\mathrm{d}}$, which is not the lifetime of the fluctuation. $\tau_{\mathrm{d}}$ is the decay time seen by one radial channel. If the fluctuating cells move, the correlation decays faster than corresponding to their lifetime. To take the spatial propagation into account, it is necessary to use the full extent in $\tau$ direction of the 1 /e contour. This is achieved by determining the intersections of the line $g$, which connects the maxima of $\varphi$ for fixed time lags $\tau$, with the $1 /$ e contour.

Similarly, one can determine the radial velocity $v_{r}^{*}$ by calculating the slope of the same line $g$ (see figure $2(b)$ ).

The interpretation of $v_{r}^{*}$, however, demands special care. As we use a diagnostic with neither poloidal nor toroidal resolution, we cannot directly determine the shape of the fluctuating cells. Therefore, the observed radial velocity can be the result of a projection onto the radial axis. An example for such a projection is shown in figure 3. The observed structure is inclined in the radial-poloidal plane and its velocity $v_{\theta}$ is directed in poloidal direction $\theta$. As the structure moves poloidally (upward), the maximum that is detected by the set of radial channels

(a)

(b)
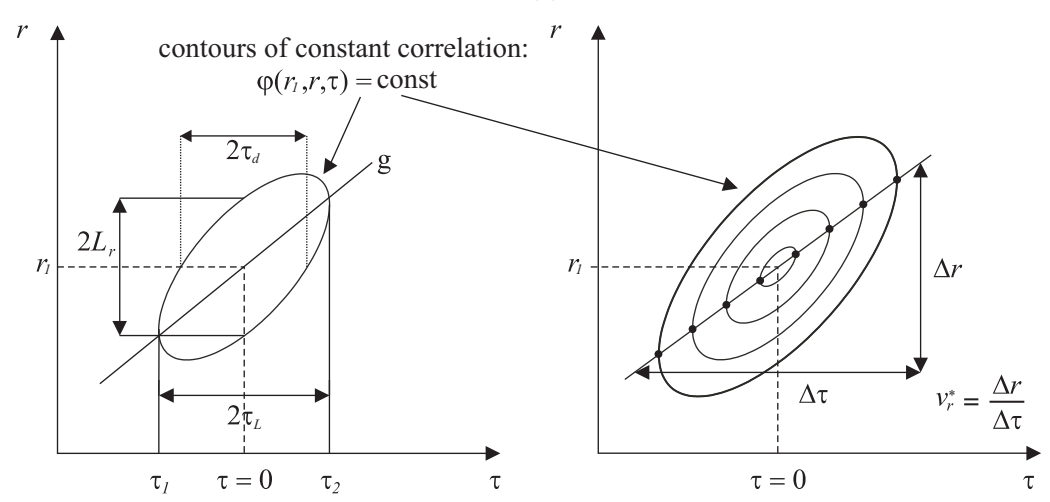

Figure 2. Determination of (a) the lifetime $\tau_{\mathrm{L}}$ and the radial correlation length $L_{r}$ and $(b)$ the observed radial velocity $v_{r}^{*}$ of the correlation function.

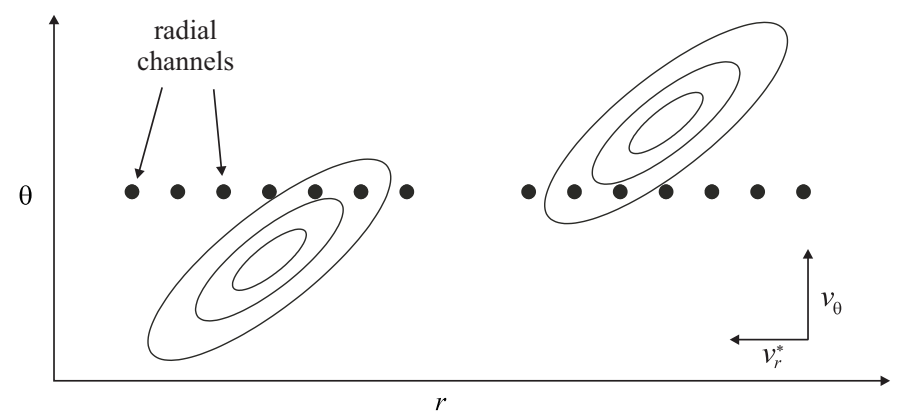

Figure 3. The radial velocity measured by a diagnostic that has only a radial resolution can be the result of the poloidal movement of the structure that is inclined in the radial-poloidal plane. 


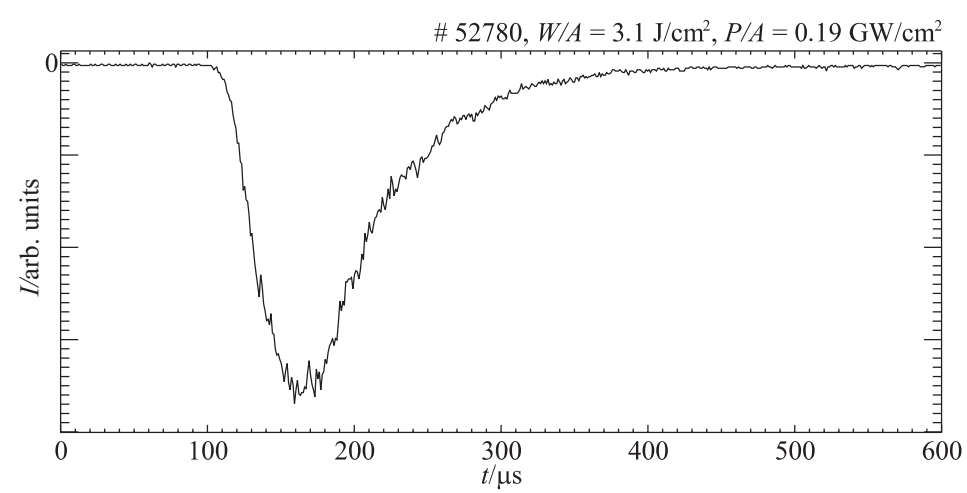

Figure 4. Example of the time trace of a photomultiplier channel.

moves radially, giving the impression of a radial movement with the velocity $v_{r}^{*}$ that does not correspond to the movement of the structure. Measurements that have been executed with an angular array of Langmuir probes on W7-AS show that such effects can indeed dominate the velocity that is derived from diagnostics with only radial resolution [32,33].

\subsection{Separation between plasma fluctuations and beam effects}

Figure 4 shows the time trace of one photomultiplier channel. As one can see, the signal depends strongly on time, as the density of Li atoms in the beam varies. To investigate the influence of plasma fluctuations, one has to find a means to eliminate the influence of the temporal variation of the Li flux on the signal.

To do so, we have made an a priori assumption that the Li flux is a sufficiently smooth function of time and can therefore be approximated by a smooth function. This assumption, however, has to be justified by the results (see section 5). Thus, we fit a smooth function $f(t)$ to the raw signal and assume that it represents the Li flux. We generally used the function

$$
f(t)=-A \mathrm{e}^{-B(t+C)}+D_{2} t^{2}+D_{1} t+D_{0},
$$

where $A, B, C$ and $D_{i=0, \ldots, 2}$ are the fitting parameters.

Figure 5(a) shows a part of the time trace of one signal together with the fitted function $f$ which we shall call baseline. If this baseline represents the variation of the Li flux, one can obtain a signal that is independent of the Li flux by subtraction of the constant background and subsequent division of the resulting signal by its baseline. To obtain the fluctuations (which by definition have a time average of 0 ), we finally subtracted the mean value-which is now close to 1 -and obtained the curve shown in figure $5(b)$.

As the statistical noise rises strongly in the last half of the time trace shown in figure 5(b), we mostly used short time traces of $110 \mu$ s length. In this case, a second-order polynomial can be used as baseline function, which considerably reduces the time needed for fitting and therefore the time required for the data analysis.

\subsection{Numerical tests}

To check the evaluation programs, we have carried out some numerical tests. For this purpose, we have used a time averaged (static) electron density profile and added some fluctuation events with a Gaussian distribution of radial velocity $v_{r}$, lifetime $\tau_{\mathrm{L}}$ and radial correlation length $L_{r}$. Additionally, we have generated Li pulses by using a fit function of the type described in section 4.2 and adding some variations on the Li flux that were characterized by an amplitude 


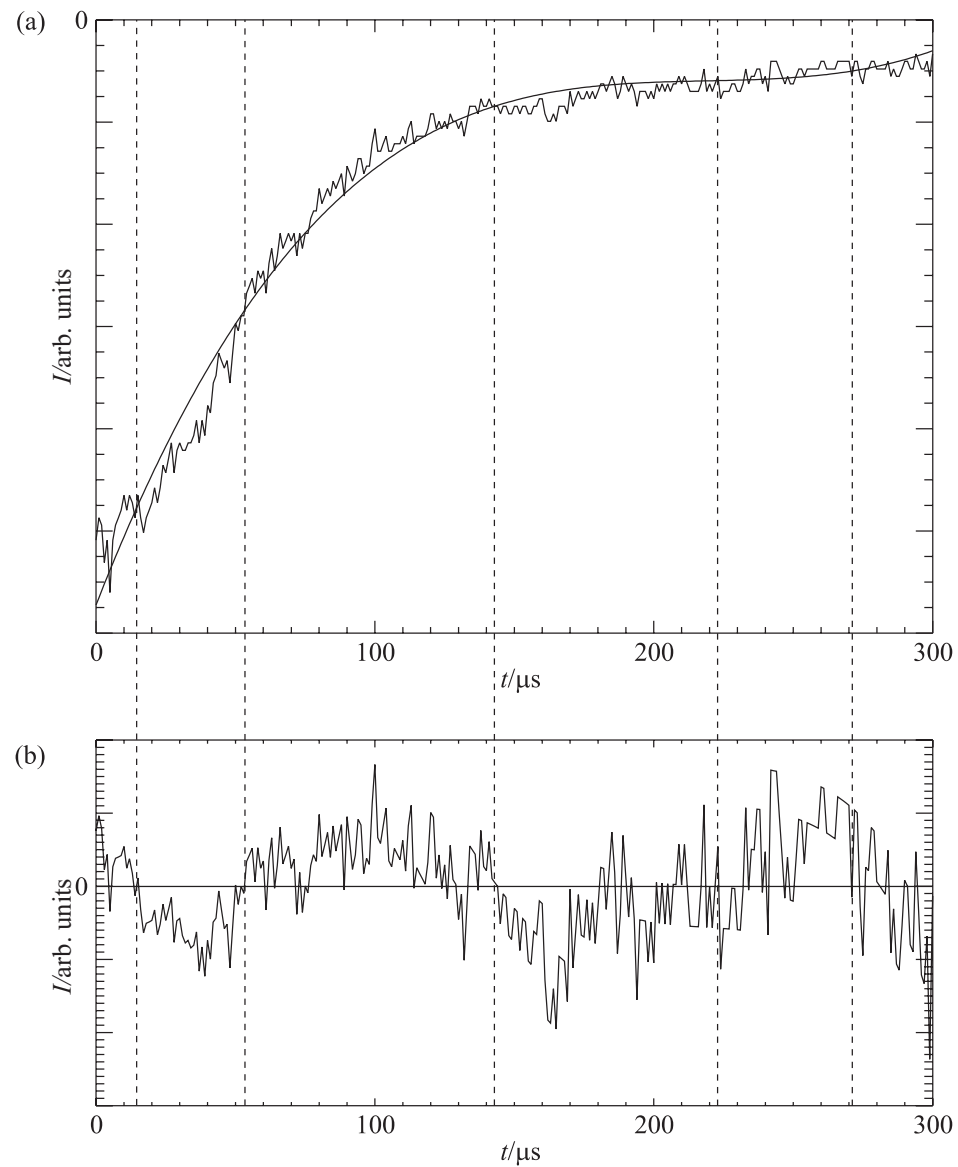

Figure 5. (a) Part of the time trace of one photomultiplier channel together with the baseline assumed to describe the variation of the Li flux and $(b)$ the same time trace normalized to the baseline.

(typically 5-10\% of the number of Li atoms in the beam) and a duration of the order of a few times $10 \mu \mathrm{s}$.

Starting from these artificially generated fluctuating electron density profiles and Li pulses, we have calculated light emission profiles according to equation (2). After adding some statistical noise, we used the artificial light emission signals at eight radial points as input for the evaluation routines to calculate the correlation functions. We then compared these correlation functions of light emission to the correlation functions calculated directly from the artificial fluctuating profiles of the electron density.

It turned out that the radial correlation length $L_{r}$ and the radial velocity $v_{r}$ of the electron density correlation functions could be reproduced with an accuracy of $10 \%$ or better. The determination of the lifetime $\tau_{\mathrm{L}}$, in contrast, can easily lead to erroneous results. If $\tau_{\mathrm{L}}$ is shorter than $10 \%$ of the length of the Li pulse, the separation between the (fast) fluctuations and the (slow) variation of the Li flux as described in section 4.2 works well. However, if $\tau_{\mathrm{L}}$ becomes comparable to the length of the Li pulses, this separation becomes impossible.

This problem is aggravated by the fact that the temporal width of correlation functions can be larger than that of the underlying fluctuations themselves: in the case of fluctuations with a Gaussian shape the width of the correlations is larger by a factor of $\sqrt{2}$. 


\section{Measurements}

\subsection{Correlation across the LCFS}

Figure 6 shows the correlation functions for four different series of comparable discharges. The time axis is the time lag $\tau$ in the definition of the correlation function (equation (6)), the spatial coordinate $z_{\mathrm{LBO}}$ is along the beam axis with the beam propagating in positive direction towards the plasma centre and $z_{0}$ gives the radial position of the reference channel. Red areas indicate a strong correlation with the reference point $\left(\tau=0 ; z_{\mathrm{LBO}}=z_{0}\right)$ and blue areas an anticorrelation. The dashed line denotes the position of the LCFS as it has been calculated with the TRANS code [34].

By variation of the vertical magnetic field $B_{z}$, the plasma was shifted inward between consecutive series. The other plasma parameters were kept constant. In all plots, the reference channel has been selected to be close to the calculated position of the LCFS.

One can see that in these discharges the areas of strong correlation outside the LCFS are tilted with respect to the axis. Inside the LCFS, however, these structures are oriented parallel to the axis. It is clearly visible that the 'turning point' where structures change from an inclined orientation to an orientation parallel to the axis is shifted together with the calculated position of the LCFS. The inclination in this representation of the correlation function indicates an apparent radial movement of the fluctuation structures and can be caused by a radial or a poloidal movement of the structures (see section 4). Correspondingly, the change of orientation

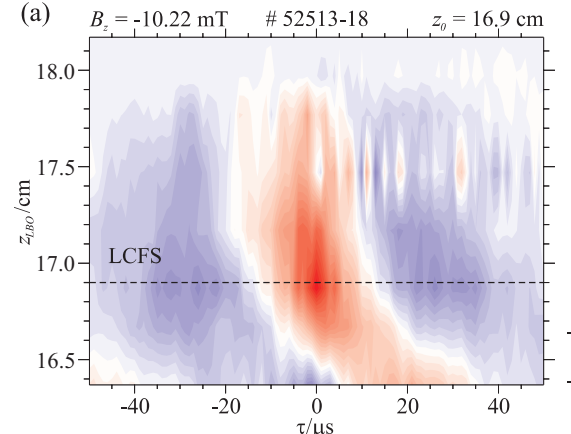

(c) $\quad B_{z}=-16.24 \mathrm{mT} \quad \# 52529-32 \quad z_{0}=17.5 \mathrm{~cm}$

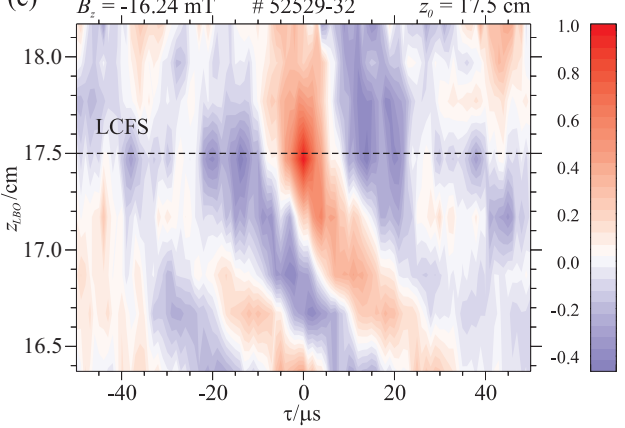

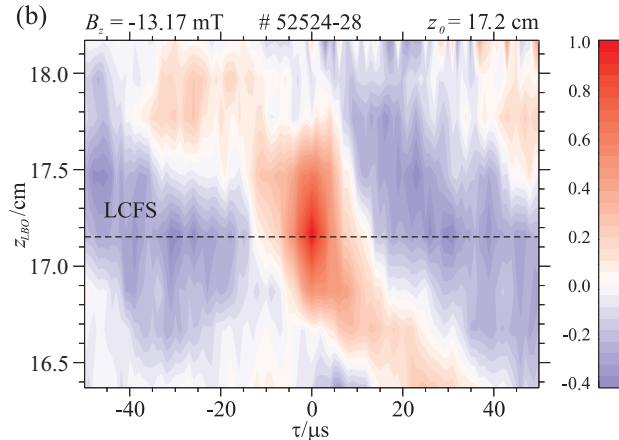

(d) $\quad B=-19.06 \mathrm{mT} \quad \# 52533-37 \quad z_{0}=17.8 \mathrm{~cm}$

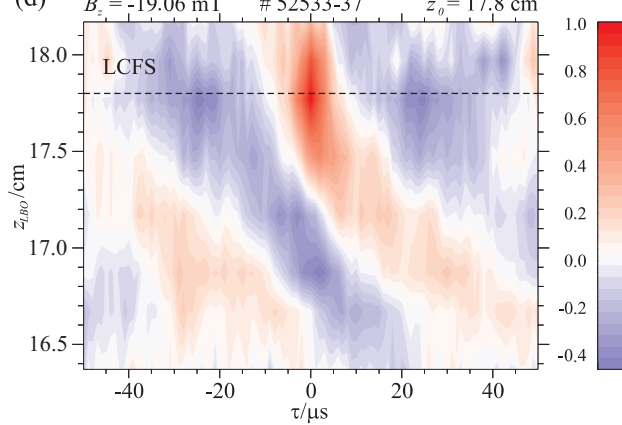

Figure 6. The normalized correlation functions for four different series of W7-AS discharges with the same line integrated electron density $\int n \mathrm{~d} l=3 \times 10^{19} \mathrm{~m}^{-2}$ (this corresponds to a line averaged electron density of $\bar{n}=5.8 \times 10^{19} \mathrm{~m}^{-3}$ ). By variation of the vertical magnetic field $B_{z}$, the plasma has been shifted inward between successive series. (The vertical magnetic field $B_{z}$ is perpendicular to the horizontal diagnostic axis $z_{\mathrm{LBO}}$.) 


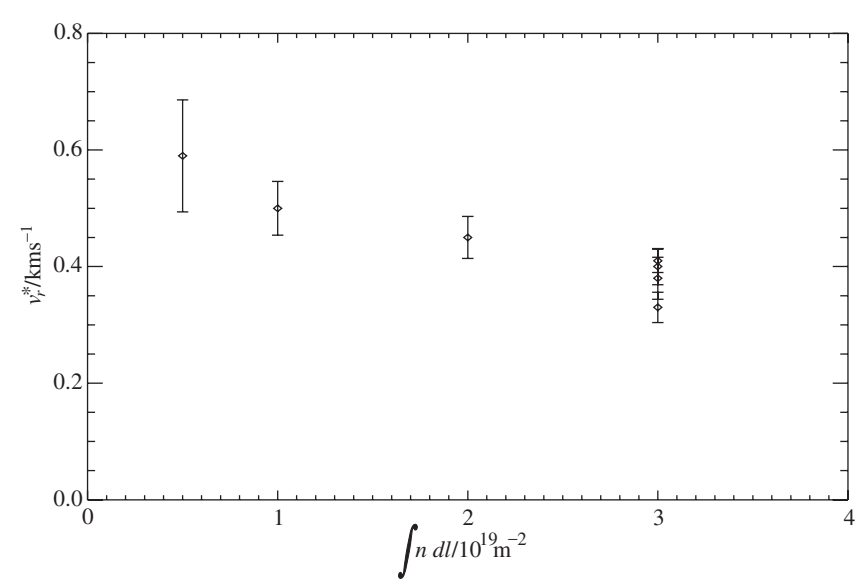

Figure 7. Dependence of the observed radial velocity $v_{r}^{*}$ on the line integrated electron density $\int n \mathrm{~d} l$ (the line averaged electron densities vary from $10^{19}$ to $5.7 \times 10^{19} \mathrm{~m}^{-3}$ ). To calculate $v_{r}^{*}$, we have determined for times $\tau_{i}$ the position $z_{\max , i}$ of the maximum of the normalized correlation function $\varphi$. In a region around the reference point $\left(\tau=0, z_{0}\right)$ the points $\left(\tau_{i} ; z_{\max , i}\right)$ have a constant slope and one can determine $v_{r}^{*}$ and its standard deviation by linear regression.

may be caused by a change in poloidal velocity. It has already been reported that the poloidal velocity reverses its sign at the LCFS $[3,35]$. We can see a change in inclination but no reversal. The reason for this can be the limited penetration depth of the Li beam.

\subsection{Influence of the electron density}

Comparing the correlation functions of discharges with different densities, one finds that the inclination angle of the structure outside of the LCFS is not always the same. One can find a dependence of the angle and the derived radial velocity $v_{r}^{*}$ on the line integrated electron density $\int n \mathrm{~d} l$ as shown in figure 7 . Obviously, the observed radial velocity $v_{r}^{*}$ decreases significantly with rising electron density.

This and the fact that the velocities measured here are much smaller than those of the atoms in the beam $\left(\sim 10^{4} \mathrm{~m} \mathrm{~s}^{-1}\right)$ show that we really measure plasma fluctuations and not a mere beam effect. Hence, our a priori assumption of a sufficiently smooth dependence of the Li flux on time is justified (see section 4). Another argument to confirm this is the dependence of the observed structures on the main magnetic field (see below).

\subsection{Influence of the direction of the magnetic field}

Figure 8 shows the correlation functions for two series of comparable discharges with opposite directions of the main magnetic field $B_{0}$. One can easily see that the observed radial velocity $v_{r}^{*}$ outside the LCFS is reversed between both plots.

However, the absolute values of $v_{r}^{*}$ for the two different directions of $B_{0}$ are also different. For the example in figure $8,\left|v_{r}^{*}\right|$ in the discharges with positive $B_{0}$ is significantly larger than in the discharges with negative $B_{0}$.

A reason could be that $v_{r}^{*}$ has two velocity components $v_{r, 1}^{*}$ and $v_{r, 2}^{*}$. If one assumes that one of these components (e.g. $v_{r, 2}^{*}$ ) depends on the direction of $B_{0}$ and the other one does not, one gets two equations:

$$
\begin{aligned}
& v_{r, 1}^{*}+\left|v_{r, 2}^{*}\right|=+330 \mathrm{~m} \mathrm{~s}^{-1} \\
& v_{r, 1}^{*}-\left|v_{r, 2}^{*}\right|=-410 \mathrm{~m} \mathrm{~s}^{-1}
\end{aligned}
$$



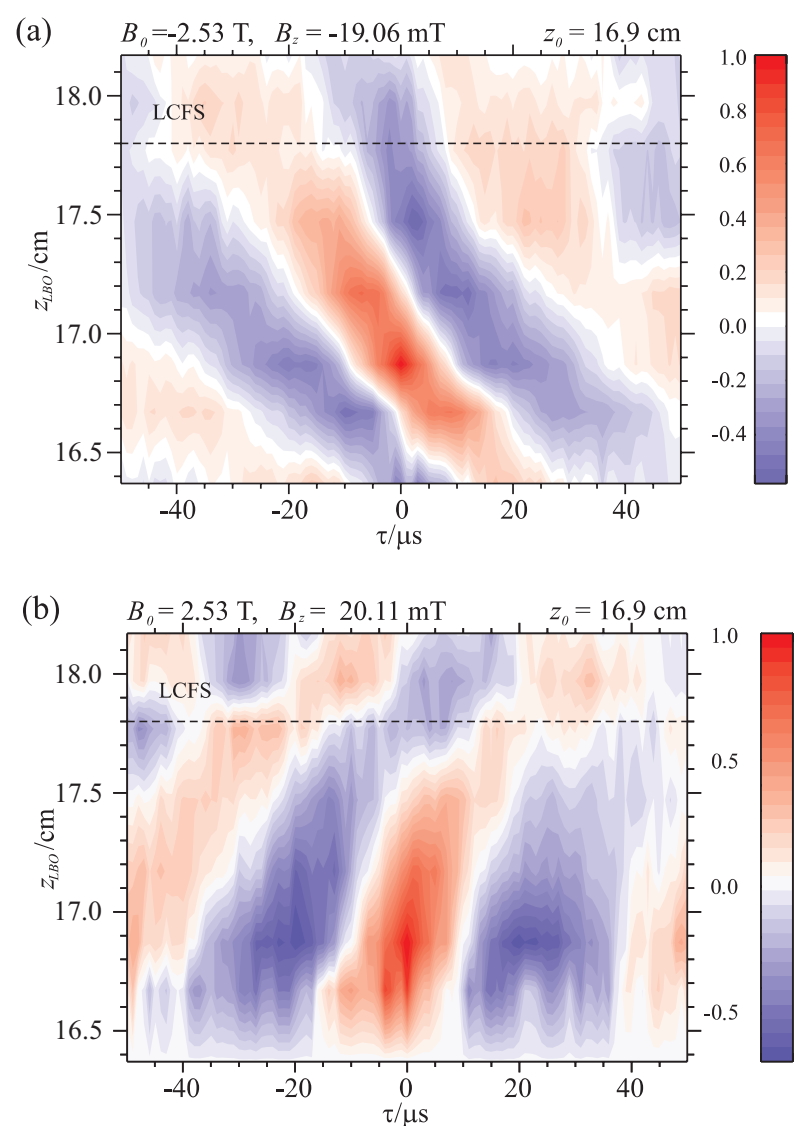

Figure 8. Comparison of the correlation functions of two series ((a) \#52533-52537 and (b) \#52941, 52942, 52946) with opposite directions of the main magnetic field $B_{0}$ but otherwise similar parameters. Linear regression leads to $(a) v_{r}^{*}=0.33 \mathrm{~km} \mathrm{~s}^{-1}, \sigma_{v}=0.023 \mathrm{~km} \mathrm{~s}^{-1}$ and (b) $v_{r}^{*}=-0.41 \mathrm{~km} \mathrm{~s}^{-1}, \sigma_{v}=0.020 \mathrm{~km} \mathrm{~s}^{-1}$.

From this, one obtains:

$$
\begin{aligned}
& v_{r, 1}^{*}=-40 \mathrm{~m} \mathrm{~s}^{-1} \\
& \left|v_{r, 2}^{*}\right|=370 \mathrm{~m} \mathrm{~s}^{-1}
\end{aligned}
$$

A possible interpretation of this result will be discussed in the following section.

\section{Discussion}

When trying to interpret the observed radial velocities $v_{r}^{*}$, it is important to keep in mind that they need not necessarily be associated with a real radial movement of fluctuation structures. Instead, the measurements presented in [32] let us assume that the observed radial velocity $v_{r}^{*}$ is largely dominated by a projection effect as described in section 4.1 . The velocities that are found here are in the same order of magnitude as the ones reported in [32]. A better agreement is not to be expected as the two diagnostics were mounted on different toroidal and poloidal positions on W7-AS and the strong variation of the plasma shape along the toroidal angle in W7-AS leads to large variations of the local poloidal flow velocity. The interpretation of the 
observed radial velocity as largely due to a projection effect would also solve the puzzle why, at magnetic field reversal, fluctuation structures should reverse their radial movement, whereas the turbulent radial $E \times B$ transport keeps its outwards direction, as it was observed in many devices.

The decreasing apparent radial velocity with increasing electron density, as depicted in figure 7 , could then be due to a decrease in poloidal velocity, a behaviour, which was already described for the electron density fluctuations in the SOL of the ASDEX tokamak [30], and which also can be found in Langmuir probe measurements of W7-AS in the electron density range $\bar{n}_{\mathrm{e}}=(1.7-7.3) \times 10^{19} \mathrm{~m}^{-3}$, although less pronounced [32, figure 17, values for $I_{\text {sat }}$ for $r=0.5, \ldots, 2 \mathrm{~cm}$ outside the LCFS].

If we, tentatively, interpret the velocity components $v_{r, 1}^{*}$ and $v_{r, 2}^{*}$ introduced in section 5.3 as due to a true radial movement $\left(v_{r, 1}^{*}\right)$ and due to the projection of a poloidal movement of inclined structures $\left(v_{r, 2}^{*}\right)$, we find that the major contribution to $v_{r}^{*}$ is not caused by a true radial movement of the fluctuation structures. This is in agreement with the observations reported in [32]. It should be stated, however, that also the poloidal velocity of the fluctuations does usually not reverse to precisely its negative value, if the magnetic field is reversed. Our tentative interpretation can therefore not replace a truly two-dimensional measurement in order to distinguish between truly radial movement and projection effect.

In [32], the authors discuss two possible mechanisms that can lead to an inclination of the fluctuating cells in the radial-poloidal plane:

- The inclination is caused by the radial shear of the poloidal $E \times B$ drift. In this case, the fluctuating cells would change their shape and inclination with time and the situation would be similar for all toroidal cross sections. Also, reversing the magnetic field would invert the inclination of the structures together with the direction of the poloidal movement (an $E \times B$ drift) and hence, the observed radial velocity $v_{r}^{*}$ would be conserved.

- The second possible reason could be the magnetic shear in conjunction with the high correlation length of the fluctuations along the magnetic field. Such an alignment of the fluctuation structures along the lines of the magnetic field would result in their inclination and shape not depending on time but on the toroidal position and the corresponding local magnetic shear. In this case, a reversal of the magnetic field would not change the orientation of the structures. Their poloidal movement, in contrast, is caused by an $E \times B$ drift and would therefore still be reversed, which would result in a reversal of $v_{r}^{*}$.

In general, both effects could act simultaneously.

The results presented in section 5.3 are evidence that the second mechanism, i.e. (local) magnetic shear, is the dominant one in generating the inclination of the fluctuation structures in the radial-poloidal plane in W7-AS. Such a behaviour is also found in three-dimensional simulations of drift-Alfvén turbulence in the edge of tokamaks (see, e.g. [36,37]).

We have investigated the magnetic field structure of the flux tubes in which the Li light was observed, performing field line tracing using the Gourdon code [38], as described in [32]. The direction of the apparent radial velocity $v_{r}^{*}$ that is expected from the combination of these calculations of the local magnetic shear and from the direction of the magnetic field, does indeed agree with the observed direction of $v_{r}^{*}$.

\section{Conclusions}

We have presented a repetitive Li laser blow-off system that has been used for the investigation of fluctuation phenomena in the SOL and edge of W7-AS plasmas. We have compared this technique and its range of application to other techniques that use Li beams. Even if the 
laser blow-off technique has its specific drawbacks, which are mainly caused by the short pulse length and strong dependence of the $\mathrm{Li}$ flux on time, it can be used for fluctuation measurements. In particular, the latter problem can be solved by normalizing the signals to the Li flux. The major advantage of the laser blow-off beam is the optimal compromise between penetration depth and spatial resolution with regard to the typical radial correlation lengths found in the edge and SOL of fusion devices.

The experimental results we presented confirm earlier results, obtained with a high energy $\mathrm{Li}$ beam [9], that the LCFS is a border line for fluctuation properties. This was shown in particular for the observed radial velocity $v_{r}^{*}$. We have also investigated the dependence of the fluctuations on the electron density and on the direction of the main magnetic field. The latter measurements complement earlier experiments with a two-dimensional array of Langmuir probes, the Li laser blow-off system having the additional advantage of perturbing the plasma less than Langmuir probes. Our results enabled us to determine the origin of the inclination of fluctuation structures in the radial-poloidal plane.

The reversal of the apparent radial velocity $v_{r}^{*}$ with magnetic field reversal, which was observed before on the TEXTOR tokamak [5] can be explained by the finding that the major contribution to $v_{r}^{*}$ is a projection effect, due to the poloidal movement of inclined structures.

\section{Acknowledgments}

The authors would like to thank K v Bovert, F Kunkel, H Preiss and D Schüller for their help during the installation of the experiment on W7-AS. The advice of S Musso, D Rusbuildt and B Schweer was vital for the construction and layout of the system. M Schubert performed the field line tracing calculations with the Gourdon code.

\section{References}

[1] Durst R D, Fonck R J, Kim J S, Paul S F, Bretz N, Bush C, Chang Z and Hulse R 1993 Phys. Rev. Lett. 713135

[2] Liewer P C 1985 Nucl. Fusion 25543

[3] Wootton A J, Carreras B A, Matsumoto H, McGuire K, Peebles W A, Ritz Ch P, Terry P W and Zweben S J 1990 Phys. Fluids B 22879

[4] Bretz N 1997 Rev. Sci. Instrum. 682927

[5] Huber A, Nedospasov A V, Samm U and Schweer B 1999 J. Nucl. Mater. 266-269 546

[6] Komori A et al 1988 Nucl. Fusion 281460

[7] McCormick K, Murmann H, El Shaer M, ASDEX Team and NI Team 1984 J. Nucl. Mater. 12148

[8] Zoletnik S, Fiedler S, Kocsis G, McCormick G K, Schweinzer J and Winter H P 1998 Plasma Phys. Control. Fusion 401399

[9] Zoletnik S, Anton M, Endler M, Fiedler S, Hirsch M, McCormick K, Schweinzer J and W7-AS Team 1999 Phys. Plasmas 64239

[10] Ledyankin A I, de Michelis C, Guirlet R, Hecq W, Hess W R and Monier-Garbet P 1996 Report CEA Centre d'Etudes Cadarache, Saint-Paul-lez-Durance (F) EUR-CEA-FC-1585

[11] Pospieszczyk A and Ross G G 1988 Rev. Sci. Instrum. 59605

[12] Komori A, Nagai S, Morisaki T and Kawai Y 1990 Rev. Sci. Instrum. 613787

[13] Breton C, de Michelis C, Hecq W and Mattioli M 1980 Rev. Phys. Appl. 151193

[14] Friichtenicht J F 1974 Rev. Sci. Instrum. 4551

[15] Marmar E S, Cecchi J L and Cohen S A 1975 Rev. Sci. Instrum. 461149

[16] Utterback N G, Tang S P and Friichtenicht J F 1976 Phys. Fluids 19900

[17] Bakos J S, Földes I B, Ignácz P N, Kocsis G, Szigeti J and Kovás J 1990 Opt. Commun. 74374

[18] Bakos J S, Földes I B, Ignácz P N and Kocsis G 1999 J. Appl. Phys. 691231

[19] Mattoo S K, Wirtz L, Pospieszczyk A and Schweer B 1997 Nucl. Instrum. Methods Phys. Res. B 124579

[20] Griem H R 1964 Plasma Spectroscopy (New York: McGraw-Hill)

[21] Schweinzer J, Brandenburg R, Bray I, Hoekstra R, Aumayr F, Janev R K and Winter H P 1999 At. Data Nucl. Data Tables 72239 
[22] Lennon M A, Bell K L, Gilbody H B, Hughes J G, Kingston A E, Murray M J and Smith F J 1988 J. Phys. Chem. Ref. Data 171285

[23] Pospieszczyk A 1993 Report: Workshop on the Use of Atomic Beams in Plasma Experiments KFKI-1993-19/D Report

[24] Huber A 1997 PhD Thesis Heinrich-Heine-Universität Düsseldorf, ISSN 0944-2952

[25] Kálvin S, Kocsis G, Bakos J S, Könen L, Mank G and Pospieszczyk P 1996 Phys. Plasmas 31324

[26] Post D E and Jensen R V 1977 At. Data Nucl. Data Tables 20397

[27] Pospieszczyk A and Ross G G 1988 Rev. Sci. Instrum. 591491

[28] Pospieszczyk A et al 1989 J. Nucl. Mater. 162-164 574

[29] Carreras B A et al 1999 Phys. Plasmas 64615

[30] Endler M, Niedermeyer H, Giannone L, Holzhauer E, Rudyj A, Theimer G, Tsois N and ASDEX Team 1995 Nucl. Fusion 351307

[31] Bendat J S and Piersol A G 2000 Random Data: Analysis and Measurement Procedures (New York: Wiley)

[32] Bleuel J, Endler M, Niedermeyer H, Schubert M, Thomsen H and the W7-AS Team 2002 New J. Phys. 438.1

[33] Bleuel J, Theimer G, Endler M, Giannone L, Niedermeyer H, ASDEX Team and W7-AS Team $199623 r d$ EPS Conf. on Controlled Fusion and Plasma Physics (Kiev) vol 20C (Geneva: European Physical Society) (part II) p 727

[34] Sardei F and Richter-Gloetzl M 1988 (rev. 1995) Transformation Package TRANS for W7-AS int. docum. IPP Garching

[35] Giannone L, Balbín R, Niedermeyer H, Endler M, Herre G, Hidalgo C, Rudyj A, Theimer G, Verplancke Ph and W7-AS Team 1994 Phys. Plasmas 13614

[36] Zeiler A, Biskamp D, Drake J F and Guzdar P N 1996 Phys. Plasmas 32951

[37] Scott B 2000 Phys. Plasmas 71845

[38] Gourdon C, Marty D, Maschke E K and Dumont J P 1969 Plasma Physics and Controlled Nuclear Fusion Research vol I (Vienna: IAEA) p 847 